\title{
Cardiovascular Care and Research in the Networked Era
}

\author{
Leslie A. Saxon
}

Received: 21 October 2010 / Accepted: 28 October 2010 /Published online: 9 November 2010

(C) Springer Science+Business Media, LLC 2010

There is a slick new television commercial advertising Apple's iPad. It includes a cool medical application that can be used for medical imaging. The advertisement shows a trend in medicine: in the last 2 years, there has been an explosion of growth in wireless medicine, which includes digestible smart pills, networked implantable devices, and smart phone applications. The United States is an innovator in this technology, which has the capability to connect patients and healthcare providers, empower patients (akin to the way the Internet changed information flow), and create a vast health network that can lead to faster cures. Investment in wireless medicine is an important way for the United States to continue its lead as a healthcare innovator.

To continue this innovation, and to have a larger and more profound global impact, the United States needs to let developers innovate; the popular sentiment that medical technology is expensive needs to be re-examined. More importantly, we need to harness our technologic investments to help the world's poor.

Developed countries spend almost $75 \%$ of costs on chronic disease management. For example, millions of Americans live with a chronic condition called heart failure, the most common cause of hospital admission for Medicare recipients. Heart failure and repeated heart failure-related hospitalizations represent a huge cost to our healthcare system. Patients discharged after treatment for heart failure face a very high risk of re-admission in the first 6 months. A cell phone or smart phone that receives information from simple sensors, that are implanted or worn inconspicuously

\footnotetext{
L. A. Saxon $(\square)$

Division of Cardiovascular Medicine, Keck School of Medicine, University of Southern California,

1510 San Pablo Street, Suite 322,

Los Angeles, CA 90033, USA

e-mail: saxon@usc.edu
}

on the body, can help physicians, patients, and family members (often living far away) monitor how well their drugs and devices are working. Allowing patients and families a chance to monitor early signs of worsening heart failure, before symptoms occur, creates a much needed connectivity and dialogue or partnership between the patients/families and doctors. This type of technology drives shift in the care paradigm and has the potential to enable personalized care, early intervention, and significantly reduce the need for re-admission.

While Apple's iPad commercial suggests that adoption is rapid, the United States can be laughably slow at widely adopting mobile computing solutions. While the United States takes pride in leading the information economy through its innovations, applying and using these technology innovations at scale is another issue. In the world of medicine, new technologies typically take 15 years to be widely used. Legacy systems and high regulatory hurdles often slow things down incredibly. We cannot afford to fall behind in this manner when it comes to wireless health.

As the director of the University of Southern California's Center for Body Computing - a cross-disciplinary center that develops products, conducts translational studies, establishes predictive policy, and creatively meets the needs of patients and physicians in wireless medicine-I see new products all of the time. Many of them come from the developing world or individual application developers. Some of these apps, which can promote better health and solve healthcare problems, never leave the engineering bench. Many of the most elegant medical applications of the last 2 years come from individual iPhone/iPad developers.

For example, one product takes information from a wireless watch that collects continuous data from hospitalized patients like heart rate, blood pressure, and temperature and can continuously stream this data to an iPad or iPhone 
for physicians to review anywhere, anytime (http://www. fastcompany.com/blog/kate-rockwood/bizzy-body/latitudeapp-makes-doctors-datasexy). This allows the doctor caring for the patient to continuously "round" on the patient and provides more immediate care as the patient's condition requires. This liberation of information to the most senior physicians enables a more continuous care model and replaces the traditional early morning rounds where decisions are made for an entire 24-h period based on one review of the patients' data taken from the prior day.

Another application monitors blood sugar through an iPhone app (http://www.fastcodesign.com/1662351/bloodglucose-monitor-for-the-iphone). Applied widely in the United States, and in the developing world, these applications could save millions of lives at much lower costs.

An app that is particularly relevant to implanted medical devices is iTracyer (http://www.itraycer.com/releases.aspx), an app that has been developed to track the devices on a global scale. It allows the real-time management of inventory, automatic manufacturer, and FDA alerts and an efficient solution for tracking of LOT and Part numbers in the unfortunate event of a recall.

My vision is of a universe where you have networked medicine that becomes like many other things in our lives, a part of our daily living. In this vision, the patient, who I think is the new healthcare consumer, will exploit wireless technology to improve their outcomes. To enable this, the healthcare information stream must become accessible to the patient as well as the physician. In this issue of Journal of Cardiovascular Translational Research (JCTR), we have examples of wireless technology that are under translational investigation. For example, a prototype multisensory system was investigated in the MUSIC-Asia study (Katra et al., this issue JCTR) and monitors heart rate, thoracic impedance, and position during activities of daily living and remotely reports the results using a wireless, noninvasive, adherent medical device. The HOMEOSTASIS registry (Troughton et al., this issue JCTR) also presents the performance characteristics of the first human use of an implanted left atrial pressure (LAP) monitoring system. Success of this LAP wireless system will enable daily monitoring and the potential for personalized heart failure therapy to predict and prevent heart failure. Although these studies illustrate the challenges of translating monitoring systems to patient care (e.g., development of meaningful daily living standards and chronic device reliability in the hostile implant environment), they nevertheless demonstrate that wireless monitoring is feasible.

We live in a global economy, and we need to continue our leadership in healthcare as we enter the Healthcare Digital Age. The physician-patient model-patient comes into the doctor's office and tries to explain his or her sickness without any hard data while the physician attempts to analyze - has not changed since Hippocrates. It is easy to romanticize medicine, but the system is really outmoded in the Information Age. We can all be much more efficient, and we can use technology, much of it already in our hands, to make the system better and less expensive.

We have a responsibility to not only use our technologic might to help the weakest in our country, and others, but we need our healthcare policies to reflect the era of the Information Age, not Ancient Greece. 